\title{
miR-218 impedes IL-6-induced prostate cancer cell proliferation and invasion via suppression of LGR4 expression
}

\author{
FUJUN LI ${ }^{1,3,4}$, CHAOHUI GU ${ }^{1,3,4}$, FENGYAN TIAN ${ }^{2}$, ZHANKUI JIA ${ }^{1,3,4}$, ZHENGLEI MENG ${ }^{1,3,4}$, \\ YINGHUI DING ${ }^{1,3,4}$ and JINJIAN YANG ${ }^{1,3,4}$ \\ Departments of ${ }^{1}$ Urology and ${ }^{2}$ Pediatrics, ${ }^{3}$ Henan Institute of Urology, ${ }^{4}$ Zhengzhou Key Laboratory for \\ Molecular Biology of Urological Tumor Research, The First Affiliated Hospital of Zhengzhou University, \\ Zhengzhou, Henan 450052, P.R. China
}

Received May 25, 2015; Accepted June 29, 2015

DOI: $10.3892 /$ or.2016.4663

\begin{abstract}
Prostate cancer is the most common solid-organ malignancy and the second leading cause of cancer-related death in males. The oncogenic effect of leucine-rich repeatcontaining $\mathrm{G}$ protein-coupled receptor (LGR) 4 has been recognized in the formation of various types of cancers, yet its regulatory mechanism in prostate cancer is still not fully understood. Previous study has shown that LGR4 may be a new responsive gene of interleukin-6 (IL-6) in cancer progression. In the present study, we established the LNCaP-IL- $6^{+}$cell subline by long-term incubation with a low concentration of IL-6 and explored the regulatory role of miR-218, a tumorsuppressing miRNA, in IL-6-induced LGR4 expression and LNCaP-IL- $6^{+}$cell proliferation and invasion. The results showed that miR-218 expression was gradually decreased and IL-6 expression was gradually increased in the process of prostate cancer progression from normal prostate, benign prostatic hyperplasia to prostate cancer, and from LNCaP to LNCaP-IL-6 ${ }^{+}$cells. Notably, we also found that miR-218 inhibited the expression of cell cycle regulatory protein cyclin A1 and invasion-related matrix metalloproteinase-9 protein induced by IL-6, and impeded the accelerative effect of IL-6 on LNCaP-IL- $6^{+}$cell proliferation, cell cycle progression and cell invasion. Moreover, our results confirmed that
\end{abstract}

Correspondence to: Dr Jinjian Yang, Department of Urology, The First Affiliated Hospital of Zhengzhou University, 1 Jianshe East Road, Zhengzhou, Henan 450052, P.R. China

E-mail: yangjinjianhn@163.com

Abbreviations: GPCR, G protein-coupled receptor; LGR4, leucine-rich repeat-containing $G$ protein-coupled receptor 4; IL-6, interleukin-6; NP, normal prostate; $\mathrm{BPH}$, benign prostatic hyperplasia; $\mathrm{PCa}$, prostate cancer; MMP-9, matrix metalloproteinase-9; miRNAs, microRNAs; H\&E, hematoxylin and eosin; FACS, fluorescence-activated cell sorting; qRT-PCR, quantitative real-time PCR; 3'-UTR, 3'-untranslated region

Key words: miR-218, IL-6, LNCaP, proliferation, invasion
miR-218 directly targets LGR4 and modulated the PI3K/Akt and $\mathrm{Wnt} / \beta$-catenin pathways in the LNCaP-IL- $6^{+}$cells. Taken together, these data clearly demonstrated the involvement of the miR-218/LGR4 regulatory pathway in IL-6-induced cell proliferation and invasion in LNCaP-IL- $6^{+}$cells via PI3K/ Akt and Wnt/ $\beta$-catenin signaling, providing new insight into therapeutics for inflammation-induced prostate cancer.

\section{Introduction}

Prostate cancer (PCa) is the most common solid-organ malignancy and the second leading cause of cancer-related death in males (1). This heterogeneous neoplasm has been reported to be primarily regulated by androgenic hormones and influenced by dietary habits (2). Chronic inflammation causes $20 \%$ of human cancers and increases the risk of cancer, including gastric, pancreatic, colon and lung cancer $(3,4)$. Increasing epidemiological and histopathological evidence also suggests that the incidence of $\mathrm{PCa}$ is correlated with inflammation, yet the concrete mechanism involved, particularly in human studies, is still not fully understood $(4,5)$. Basically, chronic inflammation creates a milieu rich in pro-inflammatory cytokines and growth factors that may lead to an uncontrolled proliferative response and genetic mutations of rapidly dividing cells $(6,7)$. Thus, PCa is closely associated with inflammation, particularly with the role of inflammatory factors.

Interleukin-6 (IL-6) is a multifunctional pro-inflammatory cytokine whose expression and function are altered in a variety of human cancers. IL- 6 has also been found to be widely expressed in clinical specimens obtained from $\mathrm{PCa}$ patients and in several PCa cell lines (2). G protein-coupled receptor (GPCR) 48, also known as leucine-rich repeat-containing GPCR (LGR) 4, is a glycoprotein hormone receptor belonging to the GPCR superfamily (8) that plays an important role in the development of multiple organs (9). In general, LGR4 encodes one of the GPCRs for R-spondins (10). A recent study found that IL-6 enhanced the expression of LGR4 protein in osteosarcoma cells, suggesting that LGR4 may be a novel responsive gene of IL-6 in cancer progression (11). Furthermore, previous preclinical and clinical studies have confirmed that GPCR is overexpressed in PCa tissues (12). LGR4 contributes greatly to the formation of various types of cancers, including lung, 
breast, prostate and gastric cancer, as well as hepatoma $(13,14)$. The effect of LGR4 on cancer progression has been recognized, yet the regulatory mechanism on LGR4 expression is still unclear.

microRNAs (miRNAs) are a set of endogenous small non-coding RNAs (19-22 bases in length) and are used to regulate gene expression by inhibiting translation or cleaving RNA transcripts in a sequence-specific manner (15). Cumulative evidence indicates that miRNAs regulate diverse biological processes, including cell proliferation, invasion, migration and apoptosis (16). miRNAs downregulate multiple target genes, including oncogenes and tumor-suppressor genes, while some miRNAs function as tumor suppressors and others act as oncogenes (17). In particular, miR-218, a tumor-suppressing miRNA, has been extensively studied in several types of cancers (18-21) and is highly downregulated in PCa $(22,23)$. In the present study, we demonstrated that LGR4 is a downstream target of miR-218 and that miR-218 inhibited PCa cell proliferation and invasion through suppressing LGR4 expression.

\section{Materials and methods}

Tissue samples and cell culture. Clinical specimens, including 51 tumor-adjacent normal prostate (NP), 56 benign prostatic hyperplasia (BPH) and $58 \mathrm{PCa}$ tissue samples from prostatectomy, were originally obtained from patients at the First Affiliated Hospital of Zhengzhou University. The specimens were maintained at $-80^{\circ} \mathrm{C}$ and then prepared for hematoxylin and eosin (H\&E) staining, quantitative real-time PCR (qRT-PCR), and western blot analyses following institutional review board approval and the patient consent.

Human LNCaP and HEK293 cell lines were purchased from the American Type Culture Collection (ATCC; Manassas, VA, USA). LNCaP cells were maintained in RPMI-1640 medium supplemented with $10 \%$ heat-inactivated fetal bovine serum (FBS) (both from Gibco, Rockville, MD, USA), $100 \mathrm{U} / \mathrm{ml}$ penicillin and $100 \mu \mathrm{g} / \mathrm{ml}$ streptomycin (Life Technologies, Rockville, MD, USA) in a humidified atmosphere of $5 \%$ $\mathrm{CO}_{2}-95 \%$ air at $37^{\circ} \mathrm{C}$. LNCaP cells were passaged once weekly in medium containing FBS and $5 \mathrm{ng} / \mathrm{ml}$ rhIL-6 (human recombinant IL-6; R\&D Systems, Minneapolis, MN, USA). After 20 passages, the new LNCaP subline was established and named 'LNCaP-IL- 6 ', and these cells were characterized until passage 73 (24). During treatment with $25 \mathrm{ng} / \mathrm{ml}$ exogenous IL-6, LNCaP-IL-6 ${ }^{+}$cells were cultured for $24 \mathrm{~h}$ without supplementation with $5 \mathrm{ng} / \mathrm{ml}$ IL-6 during routine culture. HEK293T cells were grown in Dulbecco's modified Eagle's medium (DMEM) (Gibco) supplemented with high glucose, L-glutamine, sodium pyruvate (Life Technologies) and 10\% FBS.

Bromodeoxyuridine (BrdU) assay. Cultured cells $\left(5 \times 10^{3}\right)$ after transfection or not were seeded in 96-well plates (Corning Inc., Corning, NY, USA) with $0.1 \mathrm{nM}$ synthetic androgen methyltrienolone (R1881; 17 $\beta$-hydroxy-17 $\alpha$-methylestra-4,9,11trien-3-one; New England Nuclear, Dreieichenhain, Germany) treatment in the absence or presence of $25 \mathrm{ng} / \mathrm{ml} \mathrm{IL-} 6$ for $24 \mathrm{~h}$. A cell proliferation enzyme-linked immunosorbent assay (ELISA; BrdU kit; Beyotime, Shanghai, China) was used to analyze the incorporation of BrdU during DNA synthesis according to the manufacturer's instructions. All of the experiments were performed in triplicate, and the absorbance was measured at $450 \mathrm{~nm}$ by a microplate reader (Model 450; Bio-Rad, Hercules, CA, USA).

Cell cycle analysis. Cultured cells $\left(4 \times 10^{5}\right)$ in 6 -well plates (Corning Inc.) after transfection or not were treated with $0.1 \mathrm{nM}$ R1881 in the absence or presence of $25 \mathrm{ng} / \mathrm{ml} \mathrm{IL-6.}$ After $24 \mathrm{~h}$ of incubation, the cells were collected, fixed in $70 \%$ ethanol overnight at $4^{\circ} \mathrm{C}$, stained with propidium iodide (PI; $50 \mu \mathrm{g} / \mathrm{ml})$ and DNase-free RNase A $(100 \mu \mathrm{g} / \mathrm{ml})$ (both from Sigma, St. Louis, MO, USA), and then incubated at $37^{\circ} \mathrm{C}$ for $30 \mathrm{~min}$ in the dark. Quantitative analysis of the DNA content was performed on a fluorescence-activated cell sorting (FACS)Calibur flow cytometer (Becton-Dickinson Immunocytometry Systems, San Jose, CA, USA), and the fraction of the cell population in each phase of the cell cycle was determined by flow cytometric analysis.

Transwell assay. An invasion assay was performed using Matrigel-coated 24-well Transwell chambers with 8.0- $\mu \mathrm{m}$ polycarbonate filters (Corning Inc.). The cultured cells $\left(1 \times 10^{5}\right)$ after transfection or not were seeded in serum-free RPMI-1640 medium in the upper chamber of each well, while the bottom chamber was filled with normal culture medium supplemented with $15 \%$ FBS. The drug was added to both the upper and the lower chambers. After $24 \mathrm{~h}$ of treatment with $0.1 \mathrm{nM}$ R1881

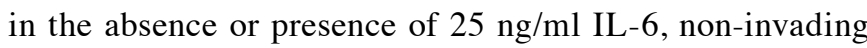
cells on the upper surface were carefully removed with a cotton-tipped swab and invading cells on the bottom surface of the filter were stained with crystal violet (Sigma). Invasion ability was quantified by counting the stained cells and statistically analyzing them.

$q R T-P C R$ assay. Total RNA of the prostate tissues or cells, including miRNAs was extracted using the miRNA isolation kit (Ambion, USA) according to the manufacturer's instructions. The purity and concentration of the RNA samples were determined using a dual-beam ultraviolet spectrophotometer (Eppendorf, Hamburg, Germany). The expression levels of mature miR-218 were analyzed using TaqMan miRNA assay (Applied Biosystems, Foster City, CA, USA), and were normalized to the expression of RNU48. The $2^{-\Delta \Delta C t}$ method was used to determine the relative quantification of miR-218 levels. The above experiment was performed in triplicate, and each assay included the negative control that lacked cDNA (25).

IL-6 determination. Frozen prostate tissue samples were sectioned into small pieces, homogenized and dissolved in RIPA lysis buffer (Beyotime). The cells were lysed and IL-6 determination was performed in triplicate via an hIL-6 ELISA kit (R\&D Systems) according to the manufacturer's instructions. The absorbance at $450 \mathrm{~nm}$ was determined using a microplate reader.

Cell transfection. hsa-miR-218 (5'-UUGUGCUUGAUCUAA CCAUGU-3'), anti-miR-218 mimics (5'-ACAUGGUUAGAU CAAGCACAA-3') and control mimics (5'-CAGUACUUU UGUGUAGUACAA-3') were synthesized by GenePharma Technology (Shanghai, China). The cultured cells were seeded 
A
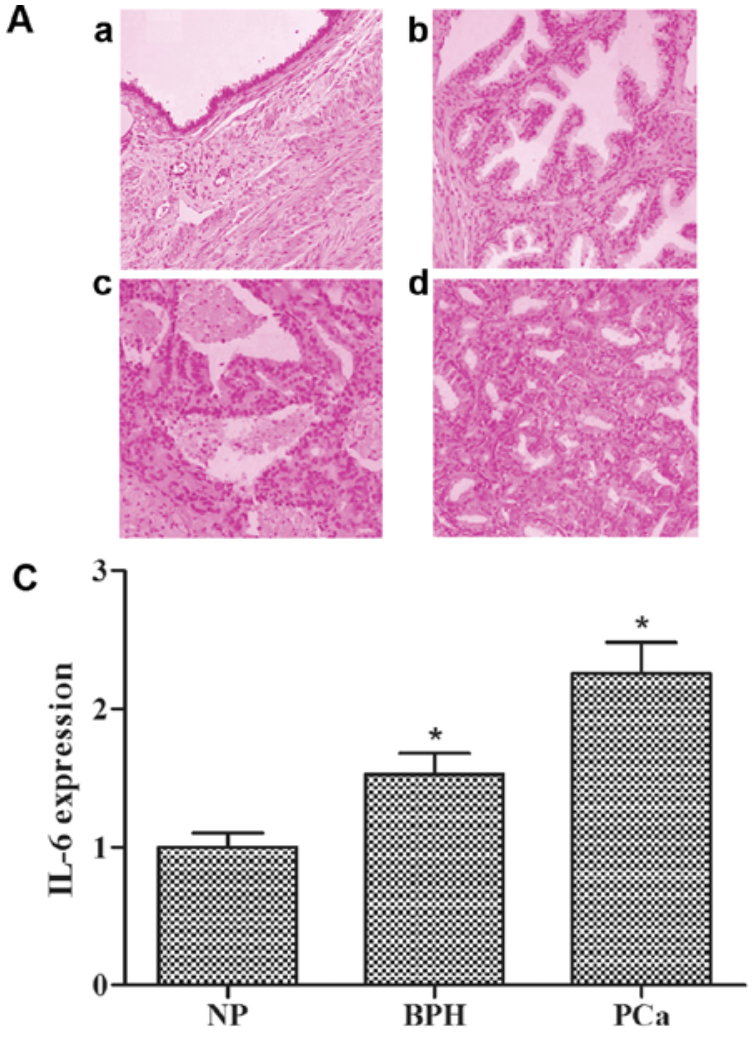

B
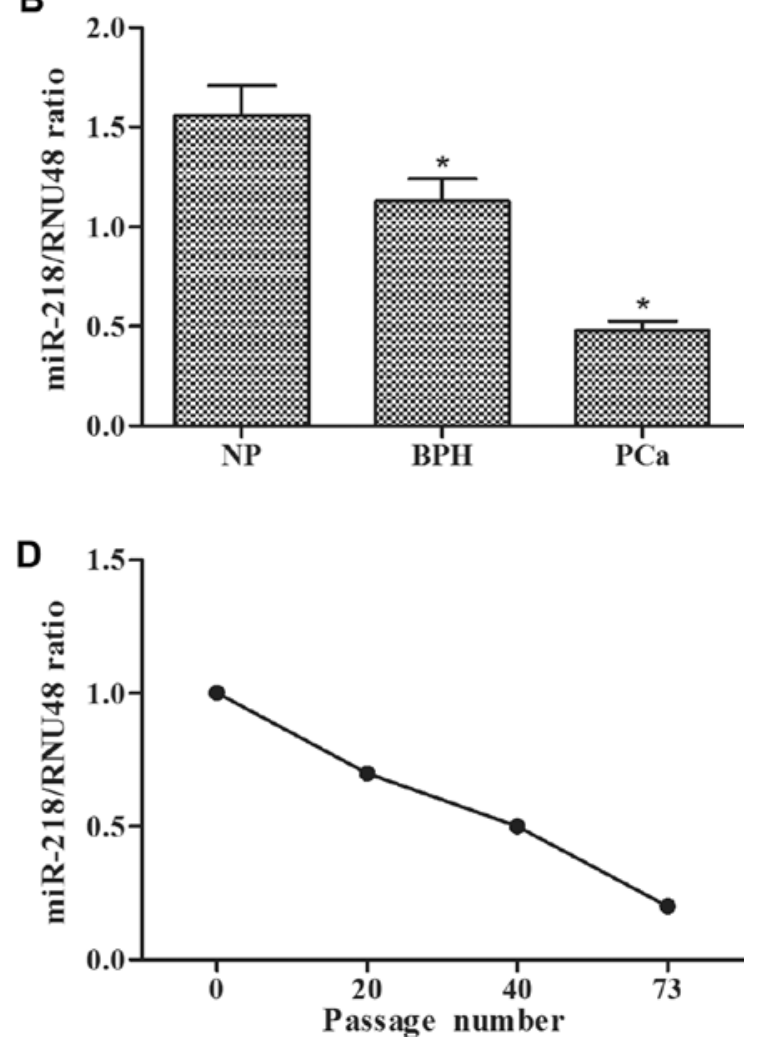

Figure 1. H\&E stainings of prostate histological structure and expression of miR-218 and IL-6 in clinical specimens and prostate cancer cells. (A) H\&E-stained images of (a) NP, (b) BPH and (c, poorly differentiated; d, well-differentiated) PCa tissue samples. Magnification, x100 of the section is shown. (B) miR-218 expression levels in clinical specimens were determined by qRT-PCR assay. (C) IL-6 expression levels in clinical specimens were determined by ELISA assay. (D) miR-218 expression levels in LNCaP and LNCaP-IL-6 ${ }^{+}$cells with increasing passage. *P<0.05 vs. the NP group. H\&E, hematoxylin and eosin; IL-6, interleukin-6; NP, normal prostate; $\mathrm{BPH}$, benign prostatic hyperplasia; $\mathrm{PCa}$, prostate cancer.

and transfected with corresponding miRNAs at working concentrations using Lipofectamime 2000 reagent (Invitrogen, Carlsbad, CA, USA) according to the manufacturer's instructions. Transfection efficiency was evaluated by qRT-PCR.

Luciferase reporter assay. The possible binding site between LGR4 and miR-218 was searched for in the microRNA database TargetScan 5.1 (http://www.targetscan.org/). The 3'-untranslated region (3'-UTR) of human LGR4 containing the miR-218 targeting sequence was inserted into the pMIRREPORT $^{\mathrm{TM}}$ miRNA expression reporter vector system (Ambion). The reporter vector plasmid containing either the LGR4-wt 3'-UTR or LGR4-mut 3'-UTR sequence was subsequently co-transfected with corresponding miRNAs into HEK293T cells using Lipofectamine 2000 reagent. The cells were harvested for luciferase assays $48 \mathrm{~h}$ after transfection. The luciferase assay kit (Promega, Madison, WI, USA) was used to measure the reporter activity according to the manufacturer's protocol.

$H \& E$ staining. The prostate tissue samples from patients were fixed in $10 \%$ formalin solution for at least $48 \mathrm{~h}$, dehydrated in alcohol, cleared in xylene and embedded in paraffin. Histological sections (4- $\mu \mathrm{m})$ were stained routinely with H\&E (hematoxylin; Fluka AG, Buchs SG, Switzerland; eosin Y, alcohol and water soluble; Winlap, UK) and then subjected to microscopic analysis.
Western blot analysis. Cell lysates were collected and protein concentrations were determined using the Pierce BCA protein assay kit (Thermo Scientific, Rockford, IL, USA). Equal amounts of protein were processed for western blotting following standard protocols. The primary antibodies used were rabbit anti-phospho-Akt polyclonal antibody (\#9271), rabbit anti-Akt polyclonal antibody (\#9272) and rabbit anti- $\beta$-catenin polyclonal antibody (\#9562) (dilution, 1:1,000; Cell Signaling Technology, Beverly, MA, USA); rabbit antiLGR4 polyclonal antibody (\#SAB4502317) (dilution, 1:1,000; Sigma); mouse anti-human cyclin A1 monoclonal antibody (\#556600) (dilution, 1:250; BD Biosciences Pharmingen, San Diego, CA, USA); mouse anti-human matrix metalloproteinase-9 (MMP-9) monoclonal antibody (sc21733) and rabbit anti- $\beta$-actin polyclonal antibody (sc130657) (dilution, 1:1,000; Santa Cruz Biotechnology, Santa Cruz, CA, USA). The resultant protein bands after incubation with a proper secondary antibody were visualized by enhanced chemiluminescence (ECL) reagent (Beyotime). The absorbance values of the target proteins were obtained through Gel-Pro Analyzer version 4.0 software (Media Cybernetics, Silver Spring, MD, USA).

Statistical analysis. Results expressed as mean \pm SD were derived from three independent experiments performed in triplicate. Statistical analysis was performed by the Student's t-test or ANOVA. $\mathrm{P}<0.05$ was considered to indicate a statistically significant result when compared to the respective control. 

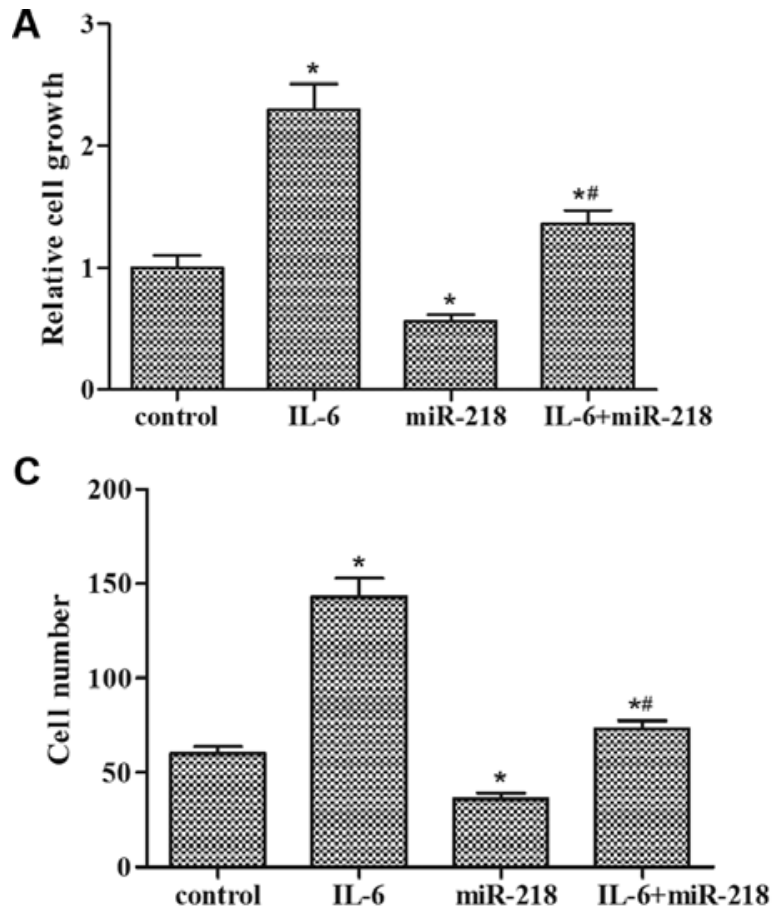

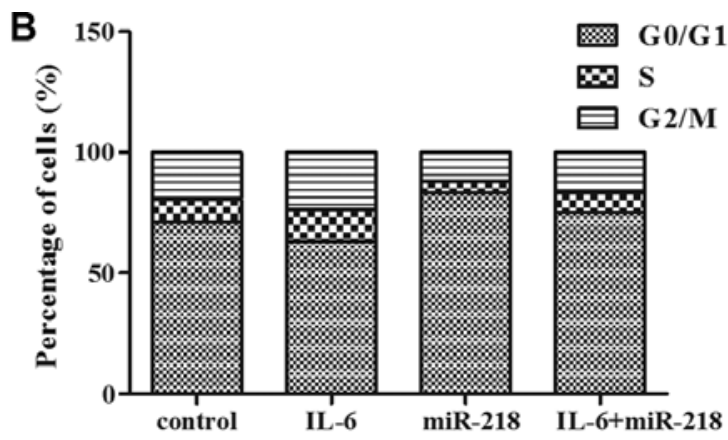

D

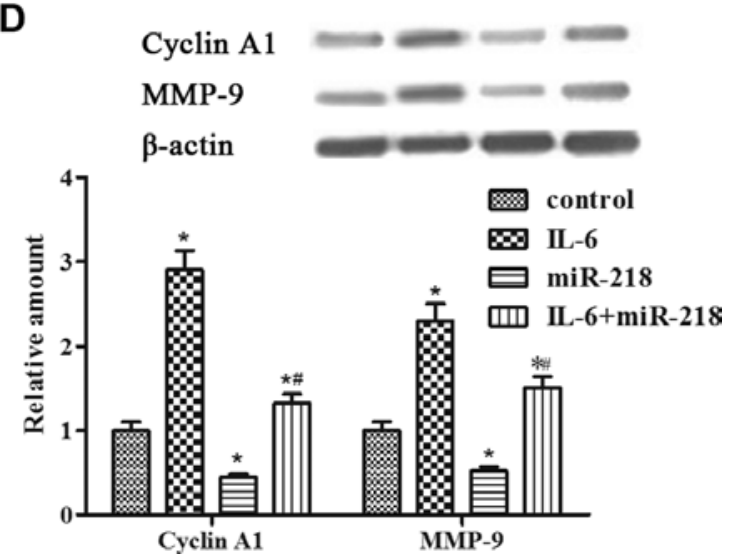

Figure 2. Effects of IL-6 and miR-218 on LNCaP-IL- $6^{+}$cell proliferation and invasion. LNCaP-IL- $6^{+}$cells after transfection or not were incubated with $0.1 \mathrm{nM}$ R1881 in the absence or presence of $25 \mathrm{ng} / \mathrm{ml} \mathrm{IL-6}$ for $24 \mathrm{~h}$ and then processed for respective detection. (A) Cell proliferation assay was performed using an ELISA BrdU kit. (B) Representative cytometric histogram of the cell cycle distribution of cells in the G0/G1, S and G2/M phases as determined by flow cytometric analysis. (C) The number of invasive cells was counted and analyzed statistically by Transwell assay. (D) The expression levels of cell cycle regulatory protein cyclin A1 and invasion-related protein MMP-9 were determined by western blotting and representative blots are shown. Quantification analysis was performed using Gel-Pro Analyzer version 4.0 software and data were normalized to $\beta$-actin. ${ }^{*} \mathrm{P}<0.05$ vs. the control group. ${ }^{\#} \mathrm{P}<0.05$ vs. the IL- 6 group. IL-6, interleukin-6; BrdU, bromodeoxyuridine; MMP-9, matrix metalloproteinase-9.

\section{Results}

miR-218 expression is downregulated and IL-6 expression is upregulated in the progression of prostate cancer. $\mathrm{H} \& \mathrm{E}$ stainings for human histological specimens were examined, and representative images of NP, BPH and PCa tissue samples are shown (Fig. 1A), in which the probable pathological progression of prostate cancer can be observed. qRT-PCR assay showed that miR-218 expression in the BPH tissues was lower than that in the NP tissues and miR-218 expression in the PCa tissues was lower when compared with that in the BPH tissues (Fig. 1B). However, ELISA assay showed that IL-6 levels in the NP, BPH and PCa tissues exhibited an opposite trend (Fig. 1C). The expression levels of miR-218 in the longterm IL-6-stimulated LNCaP cells decreased with increasing passage (Fig. 1D). These results revealed that miR-218 expression was gradually decreased and IL-6 expression was gradually increased in the process of prostate cancer progression from NP, BPH to PCa and from LNCaP to LNCaP-IL-6 ${ }^{+}$ cells.

miR-218 impedes IL-6-induced LNCaP-IL- $\sigma^{+}$cell proliferation and invasion. BrdU assay revealed that IL-6 promoted LNCaP-IL- $6^{+}$cell proliferation, whereas miR-218 transfection inhibited the enhanced cell growth (Fig. 2A). The data from FACS analysis showed that IL-6 led to a reduction in the percentage of cells in the G0/G1 phase of

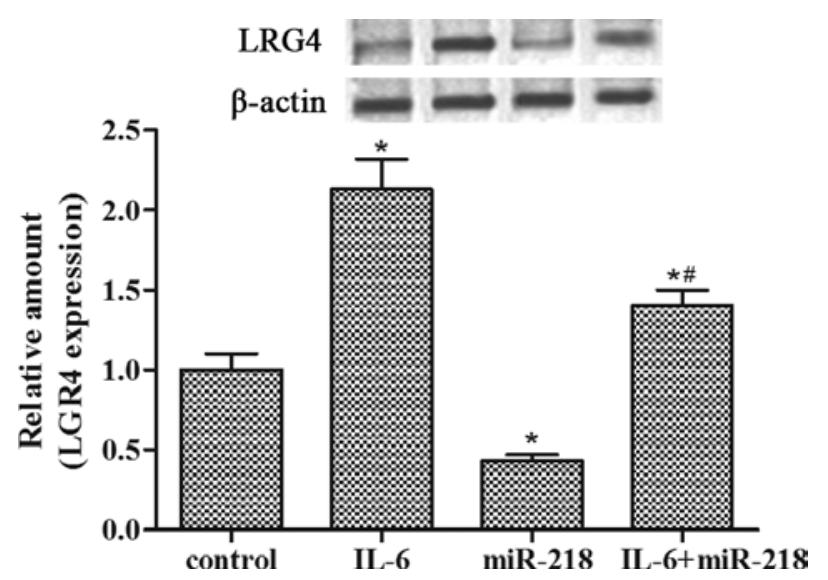

Figure 3. Effects of IL- 6 and miR-218 on LGR4 expression in the LNCaP-IL- ${ }^{+}$ cells. LNCaP-IL- $6^{+}$cells were processed as previously described. LGR4 expression was determined by western blotting and representative blots are shown. Quantification analysis was performed using Gel-Pro Analyzer version 4.0 software and data were normalized to $\beta$-actin. ${ }^{*} \mathrm{P}<0.05$ vs. the control group. ${ }^{~} \mathrm{P}<0.05$ vs. the IL-6 group. IL-6, interleukin- 6 .

the cell cycle as compared to the control group; conversely, miR-218 transfection promoted an accumulation of cells in the G0/G1 phase (Fig. 2B). Transwell assay showed that the invasive ability of the LNCaP-IL- $6^{+}$cells was significantly promoted by IL- 6 and markedly inhibited by miR-218 
A

Position 328-335 of LGR4 3' UTR binding to hsa-miR-218

$$
\begin{aligned}
& \text { (LGR4 mRNA) } \\
& \text { 5'...ACCUAUUUGUGUAUUAAGCACAA...3' } \\
& \text { 3'UGUACCAAUCUAGUUCGUGUU5' } \\
& \text { 5'...ACCUAUUUGUGUAUUGUGGUCUA...3' } \\
& \text { (LGR4 mRNA mutant) }
\end{aligned}
$$

C

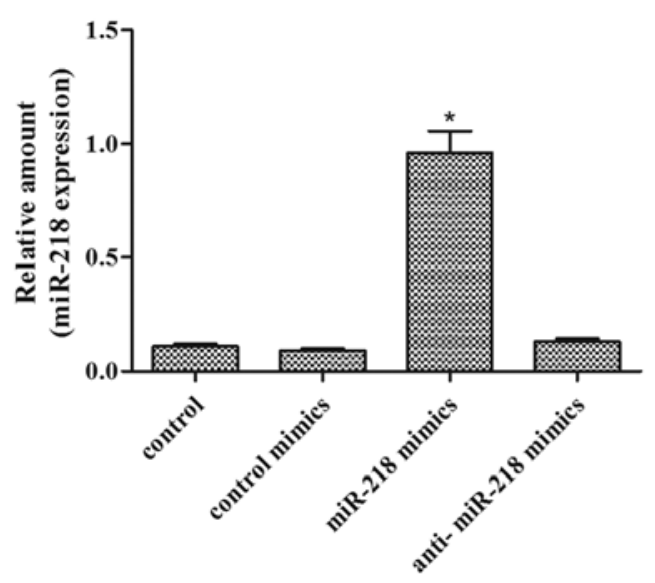

B

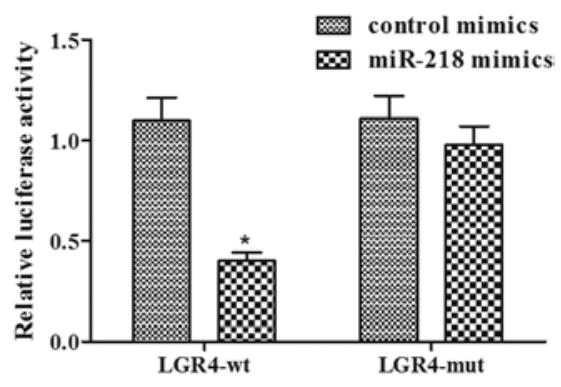

D

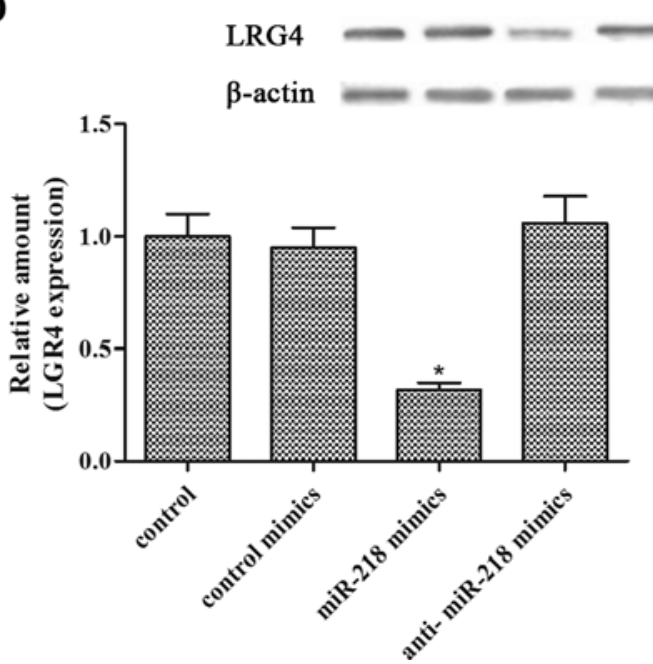

Figure 4. LGR4 is a direct target gene of miR-218. (A) TargetScan database search revealed one possible binding site in the LGR4 3'-UTR for miR-218. (B) Luciferase reporter assay was conducted in HEK293T cells. (C) miR-218 expression levels in LNCaP-IL-6 ${ }^{+}$cells were determined by qRT-PCR assay $48 \mathrm{~h}$ after transfection with the corresponding miRNAs. (D) LGR4 protein levels in the LNCaP-IL- $6^{+}$cells were determined by western blotting $48 \mathrm{~h}$ after transfection with the corresponding miRNAs. Quantification analysis was performed using Gel-Pro Analyzer version 4.0 software. ${ }^{\text {"P }}<0.05$ vs. the control group. LGR4, G protein-coupled receptor 4; 3'-UTR, 3'-untranslated region.

transfection (Fig. 2C). In addition, miR-218 transfection suppressed the increased expression of cyclin A1 and MMP-9 proteins induced by IL- 6 (Fig. 2D). Taken together, these findings indicated that miR-218 impeded IL-6-induced LNCaP-IL- $6^{+}$cell proliferation and invasion.

miR-218 inhibits IL-6-induced LGR4 expression in LNCaP-IL- $\sigma^{+}$cells. Western blotting showed that LGR4 expression was upregulated by IL- 6 pretreatment while miR-218 transfection abolished the enhanced expression of LGR4 protein induced by IL- 6 incubation in the LNCaP-IL- $6^{+}$cells (Fig. 3). These data suggest that miR-218 may be a promising candidate for directly targeting LGR4 in LNCaP-IL- $6^{+}$cells.

miR-218 targets LGR4 by binding to its 3'-UTR. Bioinformatic prediction showed that there was one putative binding site between miR-218 and the 3'-UTR of LGR4 (Fig. 4A). To confirm the binding, a luciferase reporter assay was performed by evaluating the luciferase activity of HEK293T cells transfected with the pMIR-LGR4 3'-UTR plasmids and comparing this activity with that transfected with control plasmids. The results showed that miR-218 significantly suppressed luciferase expression of LGR4-wt, whereas LGR4-mut induced no suppressive effect (Fig. 4B). In addition, qRT-PCR analysis confirmed that miR-218 transfection resulted in an increase in mature miR-218 in the LNCaP-IL- $6^{+}$cells (Fig. 4C). In addition, the LGR4 protein level was suppressed following miR-218

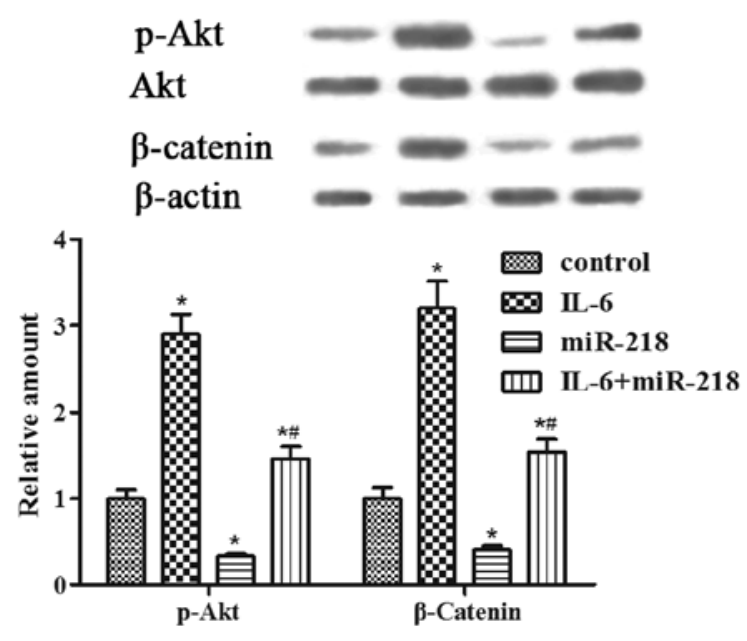

Figure 5. Effects of IL- 6 and miR-218 on the PI3K/Akt and Wnt/ $\beta$-catenin pathways in LNCaP-IL- $6^{+}$cells. $\mathrm{LNCaP}-\mathrm{IL}-6^{+}$cells were processed as previously described. The expression of Akt, p-Akt, $\beta$-catenin and $\beta$-actin proteins was detected by western blotting and representative blots are shown. Quantification analysis was performed using Gel-Pro Analyzer version 4.0 software. ${ }^{*} \mathrm{P}<0.05$ vs. the control group. ${ }^{\#} \mathrm{P}<0.05$ vs. the IL- 6 group. IL-6, interleukin-6.

transfection in the LNCaP-IL-6 ${ }^{+}$cells (Fig. 4D). In summary, these results indicated that miR-218 directly targeted LGR4 in the $\mathrm{LNCaP}-\mathrm{IL}-6^{+}$cells. 
miR-218 inhibits IL-6-induced LGR4 expression via the PI3K/Akt and Wnt/ $\beta$-catenin pathways. Furthermore, we determined whether the PI3K/Akt and Wnt/ $\beta$-catenin pathways are involved in miR-218-induced LNCaP-IL- $6^{+}$cell proliferation and invasion. Western blot analysis revealed that the levels of phospho-Akt and $\beta$-catenin expression were enhanced by IL- 6 pretreatment but were inhibited by miR-218 transfection (Fig. 5). The results indicated that miR-218 robustly suppressed the activated $\mathrm{PI} 3 \mathrm{~K} / \mathrm{Akt}$ and $\mathrm{Wnt} / \beta$-catenin pathways induced by IL- 6 in the LNCaP-IL-6 ${ }^{+}$cells.

\section{Discussion}

Prostate cancer $(\mathrm{PCa})$ is the most frequently diagnosed cancer in males, and inflammation has been associated with several diseases of the prostate, including benign enlargement and cancer (26). The expression and function of IL-6 and its receptor in PCa have also been the subject of numerous recent studies and have been investigated in human PCa cells as well as human PCa and BPH tissues obtained directly from patients $(26,27)$. miRNAs have the ability to specifically regulate multiple protein-coding genes, and bioinformatic predictions indicate that miRNAs regulate more than 30-60\% of the protein-coding genes in the human genome (28). Hence, identification of aberrantly expressed IL-6 and miRNAs is an important first step towards elucidating IL-6-mediated or miRNA-mediated oncogenic pathways.

Previous research has shown that miR-218 is significantly lower in PCa specimens and loss of tumor-suppressive miR-218 was found to enhance PCa cell invasion and migration $(22,23)$. IL-6 expression was significantly higher in PCa than in NP tissues and in patients with BPH; IL-6 expression was also elevated in patients with prostatitis compared with those without (29). Consistently, our results showed that miR-218 expression was gradually decreased and IL-6 expression was gradually increased in the process of $\mathrm{PCa}$ progression from NP, BPH to PCa and from LNCaP to LNCaP-IL- $6^{+}$cells.

miR-218 functions as a tumor-suppressing miRNA and has been extensively studied in several types of cancers $(18,20,21)$. For instance, miR-218 inhibits PCa cell proliferation and induces cell apoptosis, thus playing a tumor-suppressor role in PC3 cells (22). The effect of IL-6 on the proliferation of hormone-insensitive PCa and hormone-sensitive LNCaP cells varies under different conditions (30), yet research has shown that long-term treatment of human PCa LNCaP cells with IL-6 leads to abolishment of inhibitory growth response (24) and increased invasiveness (31). In the present study, we established the LNCaP-IL- $6^{+}$cell line by long-term incubation with a low concentration of IL- 6 and confirmed that IL- 6 promoted LNCaP-IL- $6^{+}$cell proliferation and invasion, whereas miR-218 reversed the accelerative effect of IL- 6 on cell proliferation and invasion in the LNCaP-IL- $6^{+}$cells.

LGR4 exists widely in multiple tissues and acts as a key regulator playing an important role in the process of prostate development and prostate stem cell differentiation $(14,32)$. Convincing evidence confirms that abnormal expression and activation of GPCR are intimately related to increased cancer cell proliferation, tumor growth and metastasis (12). For instance, LGR4 promotes PCa cell and tumor growth via the PI3K/Akt pathway (32), and LGR4 expression is essential for the nuclear accumulation of $\beta$-catenin in osteosarcoma cells (11). Additionally, IL-6 plays a specific role in the induction of LGR4 (11). However, miR-218 was found to inhibit AKT phosphorylation in oral cancer (33) and the downregulation of miR-218 was found to lead to stabilization and nuclear accumulation of $\beta$-catenin (34). Our results demonstrated that miR-218 targeted LGR4 by binding to its 3'-UTR, resulting in decreased LGR4 expression induced by IL-6 in the LNCaP-IL- $6^{+}$cells and downregulated the phosphorylation of PI3K/Akt and the accumulation of $\beta$-catenin.

Furthermore, cyclin A1, an important downstream target of PI3K/Akt transduced survival signals in response to IL-6 stimulation. Indeed, IL- 6 promoted cell survival by activating the PI3K/Akt pathway and increasing the expression of cyclin A1 protein in LNCaP and LNCaP-IL-6 ${ }^{+}$cells (35). In addition, IL-6 was found to upregulate MMP-9 expression to facilitate PCa cell invasion through the PI3K/Akt pathway $(36,37)$. In the present study, the results revealed that miR-218 robustly suppressed the expression of cyclin A1 and MMP-9 via the downregulation of the PI3K/Akt and Wnt/ $\beta$-catenin pathways facilitated by IL- 6 in the LNCaP-IL- $6^{+}$cells.

In conclusion, our results clearly showed the involvement of the miR-218/LGR4 regulatory pathway in IL-6-induced cell proliferation and invasion in the LNCaP-IL- $6^{+}$cells via PI3K/ Akt and $W n t / \beta$-catenin signaling. The present study elucidated the anticancer effects of miR-218 in PCa and present a novel target for $\mathrm{PCa}$ therapy.

\section{Acknowledgements}

This study was supported by the National Natural Science Foundation of China (nos. 81100464 and 81200883), the General Financial Grant from the China Postdoctoral Science Foundation (no. 2012M521410), the Foundation of Henan Educational Committee, the Scientific and Technological Innovation Project of Zhengzhou City, the Henan Natural Science Foundation, the Henan Postdoctoral Science Foundation of China, and the Youth Foundation for Medical Doctor of the First Affiliated Hospital of Zhengzhou University.

\section{References}

1. Jemal A, Siegel R, Xu J and Ward E: Cancer statistics, 2010. CA Cancer J Clin 60: 277-300, 2010.

2. Culig Z: Proinflammatory cytokine interleukin-6 in prostate carcinogenesis. Am J Clin Exp Urol 2: 231-238, 2014.

3. Coussens LM and Werb Z: Inflammation and cancer. Nature 420: 860-867, 2002

4. De Marzo AM, Platz EA, Sutcliffe S, Xu J, Grönberg H, Drake CG, Nakai Y, Isaacs WB and Nelson WG: Inflammation in prostate carcinogenesis. Nat Rev Cancer 7: 256-269, 2007.

5. Cheng I, Witte JS, Jacobsen SJ, Haque R, Quinn VP, Quesenberry CP, Caan BJ and Van Den Eeden SK: Prostatitis, sexually transmitted diseases, and prostate cancer: The California Men's Health Study. PLoS One 5: e8736, 2010.

6. Caruso C, Balistreri CR, Candore G, Carruba G, ColonnaRomano G, Di Bona D, Forte GI, Lio D, Listì F, Scola L, et al: Polymorphisms of pro-inflammatory genes and prostate cancer risk: A pharmacogenomic approach. Cancer Immunol Immunother 58: 1919-1933, 2009.

7. Klein EA and Silverman R: Inflammation, infection, and prostate cancer. Curr Opin Urol 18: 315-319, 2008.

8. Hsu SY, Liang SG and Hsueh AJ: Characterization of two LGR genes homologous to gonadotropin and thyrotropin receptors with extracellular leucine-rich repeats and a $G$ protein-coupled, seven-transmembrane region. Mol Endocrinol 12: 1830-1845, 1998 . 
9. O'Neill PR, Giri L, Karunarathne WK, Patel AK, Venkatesh KV and Gautam N: The structure of dynamic GPCR signaling networks. Wiley Interdiscip Rev Syst Biol Med 6: 115-123, 2014.

10. de Lau W, Barker N, Low TY, Koo BK, Li VS, Teunissen H, Kujala P, Haegebarth A, Peters PJ, van de Wetering M, et al: Lgr5 homologues associate with Wnt receptors and mediate R-spondin signalling. Nature 476: 293-297, 2011.

11. Liu J, Wei W, Guo CA, Han N, Pan JF, Fei T and Yan ZQ: Stat3 upregulates leucine-rich repeat-containing $\mathrm{G}$ protein-coupled receptor 4 expression in osteosarcoma cells. Biomed Res Int 2013: 310691, 2013.

12. Dorsam RT and Gutkind JS: G-protein-coupled receptors and cancer. Nat Rev Cancer 7: 79-94, 2007.

13. Zhu YB, Xu L, Chen M, Ma HN and Lou F: GPR48 promotes multiple cancer cell proliferation via activation of Wnt signaling. Asian Pac J Cancer Prev 14: 4775-4778, 2013.

14. Luo W, Rodriguez M, Valdez JM, Zhu X, Tan K, Li D, Siwko S, $\mathrm{Xin}$ L and Liu M: Lgr4 is a key regulator of prostate development and prostate stem cell differentiation. Stem Cells 31: 2492-2505, 2013.

15. Bartel DP: MicroRNAs: Genomics, biogenesis, mechanism, and function. Cell 116: 281-297, 2004.

16. Hwang HW and Mendell JT: MicroRNAs in cell proliferation, cell death, and tumorigenesis. Br J Cancer 94: 776-780, 2006.

17. Stahlhut Espinosa CE and Slack FJ: The role of microRNAs in cancer. Yale J Biol Med 79: 131-140, 2006.

18. Yang L, Li Q, Wang Q, Jiang Z and Zhang L: Silencing of miRNA-218 promotes migration and invasion of breast cancer via Slit2-Robol pathway. Biomed Pharmacother 66: 535-540, 2012.

19. Wu DW, Cheng YW, Wang J, Chen CY and Lee H: Paxillin predicts survival and relapse in non-small cell lung cancer by microRNA-218 targeting. Cancer Res 70: 10392-10401, 2010

20. Tie J, Pan Y, Zhao L, Wu K, Liu J, Sun S, Guo X, Wang B, Gang Y, Zhang Y, et al: MiR-218 inhibits invasion and metastasis of gastric cancer by targeting the Robol receptor. PLoS Genet 6 : e1000879, 2010.

21. He X, Xiao X, Dong L,Wan N, Zhou Z, Deng H and Zhang X: MiR-218 regulates cisplatin chemosensitivity in breast cancer by targeting BRCA1. Tumour Biol 36: 2065-2075, 2015.

22. Han G, Fan M and Zhang X: microRNA-218 inhibits prostate cancer cell growth and promotes apoptosis by repressing TPD52 expression. Biochem Biophys Res Commun 456: 804-809, 2015.

23. Nishikawa R, Goto Y, Sakamoto S, Chiyomaru T, Enokida H, Kojima S, Kinoshita T, Yamamoto N, Nakagawa M, Naya Y, et al: Tumor-suppressive microRNA-218 inhibits cancer cell migration and invasion via targeting of $L A S P 1$ in prostate cancer. Cancer Sci 105: 802-811, 2014.

24. Hobisch A, Ramoner R, Fuchs D, Godoy-Tundidor S, Bartsch G Klocker $\mathrm{H}$ and Culig Z: Prostate cancer cells (LNCaP) generated after long-term interleukin 6 (IL-6) treatment express IL-6 and acquire an IL-6 partially resistant phenotype. Clin Cancer Res 7: 2941-2948, 2001.

25. Gu CH, Tian FY, Pu JR, Zheng LD, Mei H, Zeng FQ, Yang JJ, Kan QC, Tong QS: Over-expression of testis-specific expressed gene 1 attenuates the proliferation and induces apoptosis of GC-1spg cells. J Huazhong Univ Sci Technolog Med Sci 34: $535-541,2014$.
26. Ben Jemaa A, Sallami S, Ramarli D, Colombatti $M$ and Oueslati R: The proinflammatory cytokine, IL-6, and its interference with bFGF signaling and PSMA in prostate cancer cells. Inflammation 36: 643-650, 2013.

27. Hobisch A, Rogatsch H, Hittmair A, Fuchs D, Bartsch G Jr, Klocker H, Bartsch G and Culig Z: Immunohistochemical localization of interleukin- 6 and its receptor in benign, premalignant and malignant prostate tissue. J Pathol 191: 239-244, 2000.

28. Friedman RC, Farh KK, Burge CB and Bartel DP: Most mammalian mRNAs are conserved targets of microRNAs. Genome Res 19: 92-105, 2009.

29. Engelhardt PF, Seklehner S, Brustmann H, Lusuardi L and Riedl CR: Immunohistochemical expression of interleukin-2 receptor and interleukin-6 in patients with prostate cancer and benign prostatic hyperplasia: Association with asymptomatic inflammatory prostatitis NIH category IV. Scand J Urol 49: 120-126, 2015.

30. Nguyen DP, Li J and Tewari AK: Inflammation and prostate cancer: The role of interleukin 6 (IL-6). BJU Int 113: 986-992, 2014.

31. Shariat SF, Chromecki TF, Hoefer J, Barbieri CE, Scherr DS, Karakiewicz PI, Roehrborn CG, Montorsi F, Culig Z and Cavarretta IT: Soluble gp130 regulates prostate cancer invasion and progression in an interleukin- 6 dependent and independent manner. J Urol 186: 2107-2114, 2011.

32. Liang F, Yue J, Wang J, Zhang L, Fan R, Zhang H and Zhang Q: GPCR48/LGR4 promotes tumorigenesis of prostate cancer via PI3K/Akt signaling pathway. Med Oncol 32: 49, 2015.

33. Uesugi A, Kozaki K-i, Tsuruta T, Furuta M, Morita K, Imoto I, Omura $\mathrm{K}$ and Inazawa J: The tumor suppressive microRNA miR-218 targets the mTOR component Rictor and inhibits AKT phosphorylation in oral cancer. Cancer Res 71: 5765-5778, 2011.

34. Lerner C, Wemmert S and Schick B: Preliminary analysis of different microRNA expression levels in juvenile angiofibromas. Biomed Rep 2: 835-838, 2014.

35. Wegiel B, Bjartell A, Culig Z and Persson JL: Interleukin-6 activates $\mathrm{PI} 3 \mathrm{~K} / \mathrm{Akt}$ pathway and regulates cyclin A1 to promote prostate cancer cell survival. Int J Cancer 122: 1521-1529, 2008.

36. Ahmad A, Sarkar SH, Aboukameel A, Ali S, Biersack B, Seibt S, Li Y, Bao B, Kong D, Banerjee S, et al: Anticancer action of garcinol in vitro and in vivo is in part mediated through inhibition of STAT-3 signaling. Carcinogenesis 33: 2450-2456, 2012.

37. Shukla S, Maclennan GT, Hartman DJ, Fu P, Resnick MI and Gupta S: Activation of PI3K-Akt signaling pathway promotes prostate cancer cell invasion. Int J Cancer 121: 1424-1432, 2007. 